\title{
Erratum to: Tert-butylhydroquinone-decorated graphene nanosheets and their enhanced capacitive behaviors
}

\section{WANG HuanWen, WU Hong Ying, CHANG YanQin, CHEN YanLi \& HU ZhongAi}

Key Laboratory of Eco-Environment-Related Polymer Materials of Ministry of Education, Key Laboratory of Polymer Materials of Gansu Province, College of Chemistry and Chemical Engineering, Northwest Normal University, Lanzhou 730070, China

Erratum to: CHINESE SCIENCE BULLETIN, July 2011 Vol.56 No.20: 2092-2097

$$
\text { doi: 10.1007/s11434-011-4424-0 }
$$

The authors report that incorrect figures were included as Figures 3 and 5 in the original publication of this paper. This error occurred during the figure editing process of the publishing company. This information should have been presented as follows:
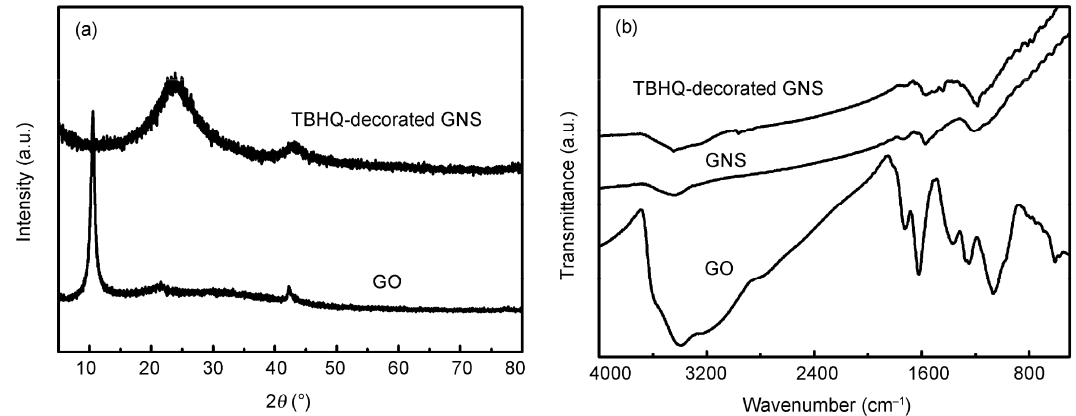

Figure 3 (a) XRD patterns of GO and the TBHQ-decorated GNS. (b) FTIR spectra of GO, GNS and the TBHQ-decorated GNS.
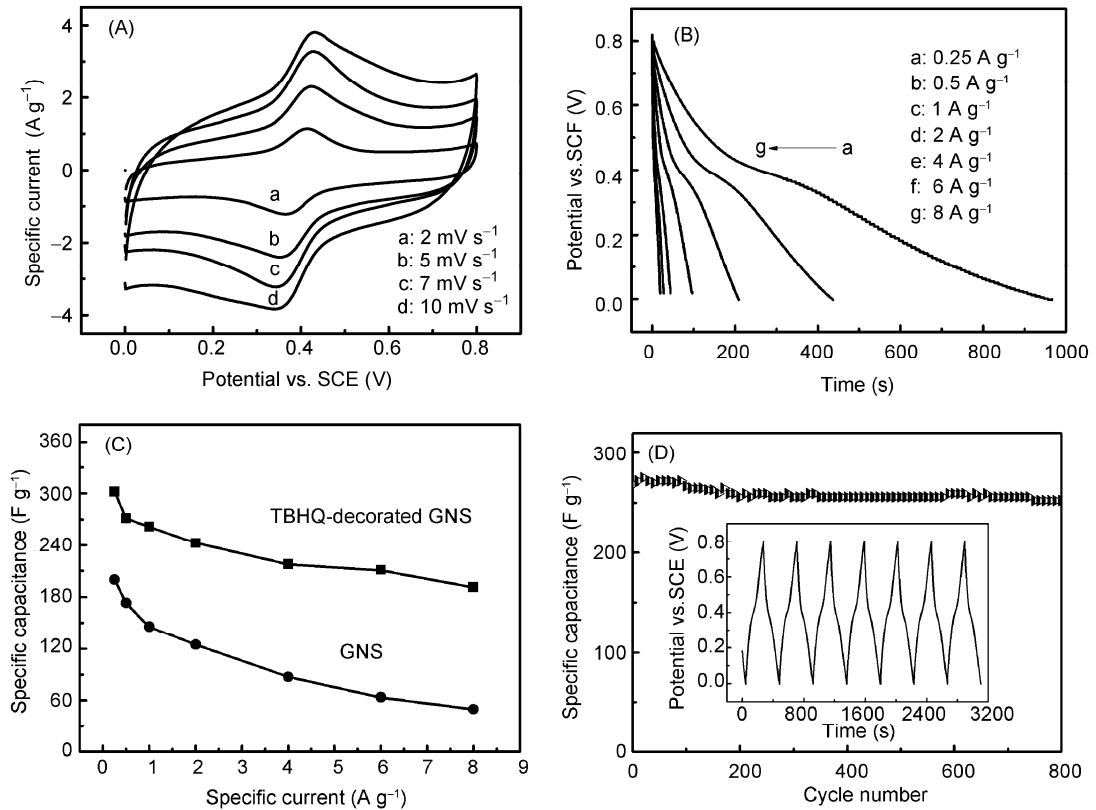

Figure 5 (A) Cyclic voltammograms of the TBHQ-decorated GNSs at different sweep rates. (B) Discharge curves of the TBHQ-decorated GNS at different specific currents. (C) Specific capacitances of the GNSs and TBHQ-decorated GNSs at different specific currents. (D) Cycle life of the TBHQ-decorated GNSs measured at $1 \mathrm{~A} \mathrm{~g}^{-1}$ and its galvanostatic charge-discharge curve (inset).

The online version of the original article can be found at http://dx.doi.org/10.1007/s11434-011-4424-0

Open Access This article is distributed under the terms of the Creative Commons Attribution License which permits any use, distribution, and reproduction in any medium, provided the original author(s) and source are credited. 